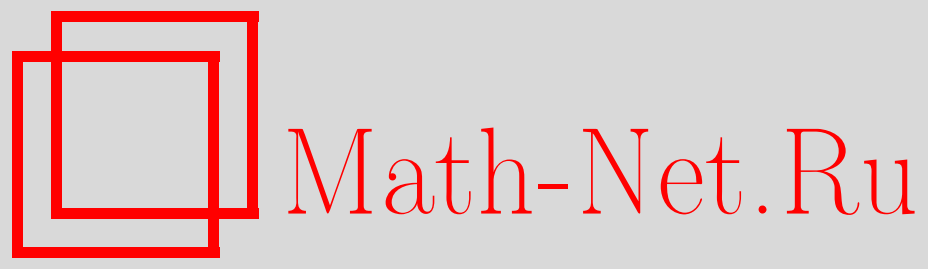

В. Ф. Молчанов, Преобразования Пуассона и Фурье для тензорных произведений, Функи. анализ и его прил., 2015, том 49, выпуск 4, 50-60

DOI: https://doi.org/10.4213/faa3211

Использование Общероссийского математического портала MathNet.Ru подразумевает, что вы прочитали и согласны с пользовательским соглашением

http://www . mathnet.ru/rus/agreement

Параметры загрузки:

IP : 54.89 .56 .158

26 апреля 2023 г., 13:53:44

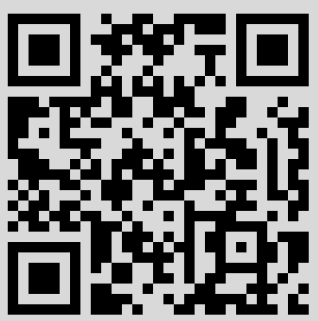


Функционалъный анализ и его приложения

2015, т. 49, вып. 4, с. 50-60

УДК 517.98

\title{
Преобразования Пуассона и Фурье для тензорных произведений*
}

\author{
(C) 2015. В. Ф. МолчАнов
}

\begin{abstract}
Для группы $G=\mathrm{SL}(2, \mathbb{R})$ мы выписываем в явном виде дифференциальные операторы, сплетающие неприводимые конечномерные представления $T_{k}$ группы $G$ с тензорными произведениями $T_{l} \otimes T_{m}$ (преобразования Пуассона и Фурье), указываем некоторый аналог гармонического анализа и приводим явные формулы для композиций этих преобразований с операторами Ли надгруппы $G \times G$. Эти построения основаны на дифференциально-разностном соотношении для ядра Пуассона.
\end{abstract}

В настоящей работе мы сообщаем несколько результатов о тензорных произведениях неприводимых конечномерных представлений группы $G=\operatorname{SL}(2, \mathbb{R})$. Хорошо известно, что тензорное произведение $T_{l} \otimes T_{m}$ раскладывается в прямую однократную сумму:

$$
T_{l} \otimes T_{m}=T_{|l-m|}+T_{|l-m|+1}+\cdots+T_{l+m-1}+T_{l+m} .
$$

Мы выписываем в явном виде операторы, сплетающие представление $T_{l} \otimes T_{m}$ и слагаемые $T_{k}$, и называем их преобразованиями Пуассона и Фурье; они двойственны друг другу. Эти преобразования оказываются дифференциальными операторами. Для преобразования Пуассона мы даем три варианта. Преобразование Фурье с точностью до множителя совпадает со скобками Ранкина-Коэна ([10], [5]).

Далее, мы записываем в явном виде формулы для композиций преобразований Пуассона и Фурье с операторами Ли надгруппы $\widetilde{G}=G \times G$ (с представлениями надалгебры). Это продолжает тематику, начатую в нашей работе [6]; см. также статьи [2], [7], [8] и библиографию в них. В этих работах мы рассматривали представления в бесконечномерных пространствах функций на однородных пространствах, сейчас мы имеем дело с конечномерными представлениями.

Побуждающим толчком для этой деятельности была работа Неретина [3] (давняя работа Мукунды [9] осталась незамеченной) для плоскости Лобачевского $\mathrm{SU}(1,1) / K$, где $K$ - диагональная подгруппа. В ней он нашел явные формулы для взаимодействия надалгебры Ли с разложением Планшереля канонического представления группы $\mathrm{SU}(1,1)$.

Мы используем другой подход к таким задачам, он не требует никакой формулы Планшереля. Для вычислений более удобным оказывается преобразование Пуассона, чем преобразование Фурье. Более того, сейчас мы видим, что в основе всей теории здесь лежит некоторое дифференциально-разностное уравнение для ядра Пуассона.

*Работа поддержана грантом РФФИ 13-01-00952-а, Госзаданием Министерства образования и науки 2014/285, проект №2476, и Региональным общественным фондом содействия отечественной науке. 
Тензорное произведение $T_{l} \otimes T_{m}$ эквивалентно представлению группы $G=$ $\mathrm{SL}(2, \mathbb{R})$ в функциях на однополостном гиперболоиде $G / H$ в $\mathbb{R}^{3}$, индуцированному характером диагональной подгруппы $H$. Однако сейчас мы не будем использовать эту реализацию представления $T_{l} \otimes T_{m}$. С другой стороны, нам кажется полезным проявить параллели с гармоническим анализом на однородных пространствах, в частности, мы вводим сферические функции.

Автор благодарит Ю. А. Неретина за полезные обсуждения.

Мы будем использовать следующие обозначения для «обобщенных степеней»:

$$
a^{[s]}=a(a+1) \cdots(a+s-1), \quad a^{(s)}=a(a-1) \cdots(a-s+1) ;
$$

здесь $a$ - число или оператор, $s \in \mathbb{N}=\{0,1,2, \ldots\}$.

\section{$\S 1$. Группа $\mathrm{SL}(2, \mathbb{R})$, ее представления}

Группа $G=\operatorname{SL}(2, \mathbb{R})$ состоит из вещественных матриц второго порядка с определителем единица:

$$
g=\left(\begin{array}{ll}
\alpha & \beta \\
\gamma & \delta
\end{array}\right), \quad \alpha \delta-\beta \gamma=1
$$

Всякое конечномерное неприводимое представление $T_{k}$ группы $G$ задается числом $k$ (старшим весом), таким, что $2 k \in \mathbb{N}=\{0,1,2, \ldots\}$. Оно действует в пространстве $V_{k}$ многочленов $\varphi(x)$ от $x$ степени $\leqslant 2 k$ (так что $\operatorname{dim} V_{k}=2 k+1$ ) по формуле

$$
\left(T_{k}(g) \varphi\right)(x)=\varphi(x \cdot g)(\beta x+\delta)^{2 k}, \quad x \cdot g=\frac{\alpha x+\gamma}{\beta x+\delta}
$$

мы считаем, что $G$ действует справа.

Алгебра Ли $\mathfrak{g}$ группы $G$ состоит из вещественных матриц второго порядка со следом 0. Базис в ней состоит из матриц

$$
L_{-}=\left(\begin{array}{cc}
0 & 0 \\
1 & 0
\end{array}\right), \quad L_{1}=\left(\begin{array}{cc}
1 / 2 & 0 \\
0 & -1 / 2
\end{array}\right), \quad L_{+}=\left(\begin{array}{cc}
0 & -1 \\
0 & 0
\end{array}\right) .
$$

Соотношения коммутации таковы:

$$
\left[L_{+}, L_{-}\right]=-2 L_{1}, \quad\left[L_{+}, L_{1}\right]=-L_{+}, \quad\left[L_{1}, L_{-}\right]=-L_{-} .
$$

Представление алгебры Ли $\mathfrak{g}$, порождаемое представлением группы $G$, мы обозначаем тем же символом, что и само исходное представление группы $G$. Для базисных элементов (1.1) из $\mathfrak{g}$ имеем

$$
T_{k}\left(L_{-}\right)=\frac{d}{d x}, \quad T_{k}\left(L_{1}\right)=x \frac{d}{d x}-k, \quad T_{k}\left(L_{+}\right)=x^{2} \frac{d}{d x}-2 k x .
$$

На пространстве $V_{k}$ существует единственная с точностью до множителя билинейная инвариантная невырожденная форма. В качестве таковой возьмем форму

$$
\langle\psi, \varphi\rangle_{k}=\frac{1}{(2 k) !} \sum_{p=0}^{2 k}(-1)^{p} \cdot \psi^{(p)}(0) \cdot \varphi^{(2 k-p)}(0),
$$

где в правой части стоят производные. Она симметрична при четном $2 k$ и кососимметрична при нечетном $2 k$ :

$$
\langle\psi, \varphi\rangle_{k}=(-1)^{2 k}\langle\varphi, \psi\rangle_{k} .
$$


Инвариантность означает, что

$$
\left\langle T_{k}(X) \psi, \varphi\right\rangle_{k}=-\left\langle\psi, T_{k}(X) \varphi\right\rangle_{k}, \quad X \in \mathfrak{g} .
$$

Для элементов базиса $\left\{x^{s}\right\}, s=0,1, \ldots, 2 k$, пространства $V_{k}$ эта форма отлична от нуля только на парах $x^{s}, x^{2 k-s}$ :

$$
\left\langle x^{s}, x^{2 k-s}\right\rangle_{k}=(-1)^{s}\left(\begin{array}{c}
2 k \\
s
\end{array}\right)^{-1} .
$$

Мы будем использовать аргументные обозначения для многочленов, находящихся внутри билинейных форм $\langle\cdot, \cdot\rangle$ : повторяющийся аргумент является немым. Например, вместо $\langle\psi, \varphi\rangle_{k}$ мы можем написать $\langle\psi(x), \varphi(x)\rangle_{k}$.

Функция $(x-\xi)^{2 k}$ есть воспроизводящее ядро (играет роль дельта-функции), а именно, для всякого многочлена $\varphi \in V_{k}$ имеет место равенство

$$
\left\langle(x-\xi)^{2 k}, \varphi(\xi)\right\rangle_{k}=\varphi(x),
$$

так что $(x-\xi)^{2 k}$ есть аналог ядра Бергмана для базиса $\left\{x^{s}\right\}, s=0,1, \ldots, 2 k$, пространства $V_{k}$. Дифференцируя (1.7), получаем

$$
\left\langle(x-\xi)^{2 k-b}, \varphi(\xi)\right\rangle_{k}=\frac{1}{(2 k)^{(b)}} \cdot \frac{d^{b} \varphi(x)}{d x^{b}} .
$$

\section{§2. Тензорные произведения}

Тензорное произведение $V_{l} \otimes V_{m}$ состоит из многочленов $f(x, y)$ от $x, y$ степени $\leqslant 2 l$ по $x$ и степени $\leqslant 2 m$ по $y$. В этом пространстве действует представление $T_{l m}=T_{l} \times T_{m}$ группы $(\ll$ надгруппы») $\widetilde{G}=G \times G$ :

$$
\left(T_{l m}\left(g_{1}, g_{2}\right) f\right)(x, y)=f\left(x \cdot g_{1}, y \cdot g_{2}\right) \cdot\left(\beta_{1} x+\delta_{1}\right)^{2 l}\left(\beta_{2} y+\delta_{2}\right)^{2 m} .
$$

В группе $\widetilde{G}$ естественно выделяются три подгруппы, изоморфные $G$. Первая из них, обозначим ее через $G^{d}$, есть диагональ, состоящая из пар $(g, g), g \in G$. Далее, имеются две компонентные подгруппы, обозначим их через $G_{1}$ и $G_{2}$, состоящие соответственно из пар $(g, E)$ и $(E, g)$, где $E$ - единичная матрица, $g \in G$.

Ограничение представления $T_{l m}$ на группу $G^{d}$ есть тензорное произведение $T_{l} \otimes T_{m}$ представлений $T_{l}$ и $T_{m}$ группы $G$ :

$$
\left(\left(T_{l} \otimes T_{m}\right)(g) f\right)(x, y)=f(x \cdot g, y \cdot g) \cdot(\beta x+\delta)^{2 l}(\beta y+\delta)^{2 m} .
$$

Положим

$$
r=m-l .
$$

Известно, что тензорное произведение $T_{l} \otimes T_{m}$ раскладывается в прямую однократную сумму:

$$
T_{l} \otimes T_{m}=\sum_{k} T_{k}
$$

где $k$ пробегает множество чисел

$$
|r|,|r|+1, \ldots, l+m-1, l+m .
$$

Соответственно этому $V_{l} \otimes V_{m}$ разлагается в сумму подпространств:

$$
V_{l} \otimes V_{m}=\sum_{k} W_{k}
$$


инвариантных и неприводимых относительно $T_{l} \otimes T_{m}$. Для облегчения записи мы не будем явно указывать зависимость от $l, m$ как здесь в правой части, так и ниже. Ограничение представления $T_{l} \otimes T_{m}$ на $W_{k}$ эквивалентно $T_{k}$.

Билинейные формы на $V_{l}$ и $V_{m}$ порождают билинейную форму $\langle f, h\rangle_{l m}$ на $V_{l} \otimes V_{m}:$

$$
\langle f, h\rangle_{l m}=\frac{1}{(2 l) !(2 m) !} \sum_{p, q}(-1)^{p+q} \cdot f^{(p, q)}(0,0) h^{(2 l-p, 2 m-q)}(0,0) .
$$

Она инвариантна относительно представления $T_{l m}=T_{l} \times T_{m}$ надгруппы $\widetilde{G}$. Подпространства $W_{k}$ ортогональны относительно билинейной формы $\langle f, h\rangle_{l m}$.

\section{§3. Преобразования Пуассона и Фурье}

Пусть $k$ принадлежит множеству (2.2). Положим

$$
j=l+m-k,
$$

так что $2 l-j=k-r, 2 m-j=k+r$, см. (2.1). Назовем ядром Пуассона следующий многочлен из $V_{l} \otimes V_{m} \otimes V_{k}$ :

$$
P_{k}(x, y ; t)=(y-t)^{2 m-j}(x-t)^{2 l-j}(y-x)^{j}=(y-t)^{k+r}(x-t)^{k-r}(y-x)^{j} .
$$

Он инвариантен относительно представления $T_{l} \otimes T_{m} \otimes T_{k}$ группы $G$ :

$$
\left(\left(T_{l}(g) \otimes T_{m}(g) \otimes T_{k}(g) P_{k}\right)(x, y ; t)=P_{k}(x, y ; t), \quad g \in G .\right.
$$

С помощью ядра Пуассона $P_{k}$ определим два отображения: преобразование Пуассона $M_{k}: V_{k} \rightarrow V_{l} \otimes V_{m}$ и преобразование Фуръе $F_{k}: V_{l} \otimes V_{m} \rightarrow V_{k}$. А именно,

$$
\begin{aligned}
& \left(M_{k} \varphi\right)(x, y)=\left\langle P_{k}(x, y ; t), \varphi(t)\right\rangle_{k}, \\
& \left(F_{k} f\right)(t)=\left\langle f(x, y), P_{k}(x, y ; t)\right\rangle_{l m} .
\end{aligned}
$$

В силу (3.3) операторы $M_{k}$ и $F_{k}$ cnлетают представления $T_{l} \otimes T_{m}$ и $T_{k}$ группы $G$, а именно,

$$
\begin{aligned}
M_{k} T_{k}(g) & =\left(T_{l} \otimes T_{m}\right)(g) M_{k}, \\
T_{k}(g) F_{k} & =F_{k}\left(T_{l} \otimes T_{m}\right)(g) .
\end{aligned}
$$

Преобразования Пуассона и Фурье сопряжены друг другу:

$$
\left\langle f, M_{k} \varphi\right\rangle_{l m}=\left\langle F_{k} f, \varphi\right\rangle_{k} .
$$

Из (1.7) следует, что эти преобразования переводят воспроизводящие ядра в пространствах $V_{k}$ и $V_{l} \otimes V_{m}$ в ядро Пуассона:

$$
\begin{gathered}
\left(M_{k}(t-\xi)^{2 k}\right)(x, y)=P_{k}(x, y ; \xi), \\
\left(F_{k}(\xi-x)^{2 l}(\eta-y)^{2 m}\right)(t)=P_{k}(\xi, \eta ; t) .
\end{gathered}
$$


Теорема 3.1. Преобразование Пуассона $M_{k}: V_{k} \rightarrow V_{l} \otimes V_{m}$ можно записать в одном из следующих трех видов (здесь $\left.\varphi \in V_{k}\right)$ :

$$
\begin{aligned}
& \left(M_{k} \varphi\right)(x, y)=\sum_{\alpha=0}^{k+r}\left(\begin{array}{c}
k+r \\
\alpha
\end{array}\right) \frac{1}{(2 k)^{(\alpha)}}(y-x)^{j+\alpha}\left(\frac{d}{d x}\right)^{\alpha} \varphi(x), \\
& \left(M_{k} \varphi\right)(x, y)=c_{k} \cdot(y-x)^{j}\left\{(y-x) \frac{d}{d x}+k-r+1\right\}^{[k+r]} \varphi(x), \\
& \left(M_{k} \varphi\right)(x, y)=c_{k} \cdot(y-x)^{l+m+k+1}\left(\frac{d}{d x}\right)^{k+r}(y-x)^{-k+r-1} \varphi(x),
\end{aligned}
$$

где $r$ и j даются формулами (2.1) u (3.1), а $c_{k}=1 /(2 k)^{(k+r)}$.

Доказательство. Докажем сначала (3.6). Заменив $y-t$ на $(y-x)+(x-t)$ в ядре Пуассона (3.2) и применив формулу бинома Ньютона, приведем это ядро к виду

$$
P_{k}(x, y ; t)=(y-x)^{j} \sum_{\alpha=0}^{k+r}\left(\begin{array}{c}
k+r \\
\alpha
\end{array}\right)(y-x)^{\alpha}(x-t)^{2 k-\alpha} .
$$

Подставив это в (3.4) и использовав (1.8), мы получим (3.6). После этого формулы (3.7) и (3.8) доказываются непосредственной проверкой.

Теорема 3.2. Преобразование Фуръе $F_{k}: V_{l} \otimes V_{m} \rightarrow V_{k}$ дается следующей формулой $\left(f(x, y)\right.$ - многочлен из $\left.V_{l} \otimes V_{m}\right)$ :

$$
\left(F_{k} f\right)(t)=\sum_{\alpha=0}^{j}\left(\begin{array}{l}
j \\
\alpha
\end{array}\right)(-1)^{j-\alpha} \frac{1}{(2 l)^{(j-\alpha)}(2 m)^{(\alpha)}} \cdot f^{(j-\alpha, \alpha)}(t, t),
$$

где ј дается формулой (3.1).

Доказательство. Теперь в ядре Пуассона (3.2) заменим $y-x$ на $(y-t)-(x-t)$ и применим формулу бинома Ньютона; тогда это ядро приведется к виду

$$
P_{k}(x, y ; t)=\sum_{\alpha=0}^{j}\left(\begin{array}{l}
j \\
\alpha
\end{array}\right)(-1)^{\alpha}(y-t)^{2 m-\alpha}(x-t)^{2 l-(j-\alpha)} .
$$

Подставив это в (3.5) и использовав (1.4) и (1.8), мы получим (3.9).

Разложим ядро Пуассона $P_{k}(x, y ; t)$ по степеням $t$ :

$$
P_{k}(x, y ; t)=(y-x)^{j} \sum_{p=0}^{2 k} N_{k, 2 k-p}(x, y)(-t)^{p},
$$

где

$$
N_{k, p}(x, y)=\sum_{\alpha+\beta=p}\left(\begin{array}{c}
k+r \\
\alpha
\end{array}\right)\left(\begin{array}{c}
k-r \\
\beta
\end{array}\right) y^{\alpha} x^{\beta} .
$$

Преобразование Пуассона $M_{k}$ переводит базис $\left\{t^{b}\right\}, b=0,1, \ldots, 2 k$, в пространстве $V_{k}$ в базис $\left\{M_{k} t^{b}\right\}$ в пространстве $W_{k}$. Из $(3.4),(1.6),(3.10)$ получаем, что

$$
\left(M_{k} t^{b}\right)(x, y)=\left(\begin{array}{c}
2 k \\
b
\end{array}\right)^{-1}(y-x)^{j} N_{k, b}(x, y) .
$$


Таким образом, ядро Пуассона $P_{k}(x, y ; t)$ служит производящей функцией для коэффициентов Клебша-Гордана (мы не заботимся о нормировке). Аналогичную производящую функцию для коэффициентов Клебша-Гордана для группы $\mathrm{SU}(2)$ можно найти, например, в [4].

Композиция $F_{k} M_{k}$ преобразований Пуассона и Фурье есть скалярный оператор в пространстве $V_{k}$ :

$$
F_{k} M_{k} \varphi=\omega_{k} \varphi, \quad \varphi \in V_{k},
$$

где

$$
\omega_{k}=\frac{j !(l+m+k+1)^{(j)}}{(2 l)^{(j)}(2 m)^{(j)}} .
$$

Следовательно, обратная композиция (с множителем) $\omega_{k}^{-1} M_{k} F_{k}$ проектирует $V_{l} \otimes V_{m}$ на $W_{k}$. Отсюда следует формула обращения:

$$
f=\sum_{k} \frac{1}{\omega_{k}} M_{k}\left(F_{k} f\right),
$$

восстанавливающая функцию $f \in V_{l} \otimes V_{m}$ по ее компонентам Фурье $F_{k} f$, и формула Планшереля для функций $f, h \in V_{l} \otimes V_{m}$ :

$$
\langle f, h\rangle_{l m}=\sum_{k} \frac{1}{\omega_{k}}\left\langle F_{k} f, F_{k} h\right\rangle_{k},
$$

а также «унитарность» преобразования Пуассона:

$$
\left\langle M_{k} \varphi, M_{k} \psi\right\rangle_{l m}=\omega_{k}\langle\varphi, \psi\rangle_{k} .
$$

Функция

$$
\Pi_{k}(u, v ; x, y)=\left\langle P_{k}(u, v ; t), P_{k}(x, y ; t)\right\rangle_{k}
$$

служит ядром оператора $M_{k} F_{k}$ :

$$
\left(M_{k} F_{k} f\right)(x, y)=\left\langle\Pi_{k}(u, v ; x, y), f(u, v)\right\rangle_{l m} .
$$

Формула обращения означает (см. (3.13) и (3.12)), что

$$
\sum_{k} \frac{1}{\omega_{k}} \Pi_{k}(u, v ; x, y)=(x-u)^{2 l}(y-v)^{2 m} .
$$

Пусть $H$ обозначает подгруппу диагональных матриц

$$
h=\left(\begin{array}{cc}
\lambda^{-1} & 0 \\
0 & \lambda
\end{array}\right) .
$$

Возьмем в $G$ матрицу $g_{x y}$, зависящую от $x, y$,

$$
g_{x y}=\left(\begin{array}{cc}
1 & -1 /(y-x) \\
-x & y /(y-x)
\end{array}\right) .
$$

Одночлены $\theta_{k}^{(-)}(t)=(-t)^{k-r}$ и $\theta_{k}^{(+)}(t)=(-1)^{2 r} t^{k+r}$ из $V_{k}$ являются собственными векторами для операторов $T_{k}(h), h \in H$, с собственными значениями $\lambda^{2 r}$, $\lambda^{-2 r}$ соответственно. Сдвиг этих одночленов с помощью матрищ $g_{x y}$ и $g_{y x}$ соответственно дает ядро Пуассона $P_{k}(x, y ; t)$, а именно,

$$
P_{k}(x, y ; t)=(y-x)^{2 m}\left(T_{k}\left(g_{x y}\right) \theta_{k}^{(-)}\right)(t)=(y-x)^{2 l}\left(T_{k}\left(g_{y x}\right) \theta_{k}^{(+)}\right)(t) .
$$


Преобразование Пуассона переводит $\theta_{k}^{( \pm)}$в некоторый многочлен $\Psi_{k}^{( \pm)}(x, y)$,

$$
\Psi_{k}^{( \pm)}=M_{k} \theta_{k}^{( \pm)} .
$$

Мы назовем многочлены $\Psi_{k}^{( \pm)}(x, y)$ сферическими функииями. Для каждого $k$ обе функции $\Psi_{k}^{(-)}(x, y)$ и $\Psi_{k}^{(+)}(x, y)$ принадлежат $W_{k}$ и являются собственными для $T_{l m}(h), h \in H$, с собственными значениями $\lambda^{2 r}, \lambda^{-2 r}$, а следовательно, однородными многочленами степеней $2 l$ и $2 m$ соответственно. Из (3.11) получаем

$$
\begin{aligned}
& \Psi_{k}^{(-)}(x, y)=(-1)^{k-r} d_{k} \cdot(y-x)^{j} N_{k, k-r}(x, y), \\
& \Psi_{k}^{(+)}(x, y)=(-1)^{2 r} d_{k} \cdot(y-x)^{j} N_{k, k+r}(x, y),
\end{aligned}
$$

где

$$
d_{k}=\left(\begin{array}{c}
2 k \\
k-r
\end{array}\right)^{-1}=\left(\begin{array}{c}
2 k \\
k+r
\end{array}\right)^{-1} .
$$

Функции $\Psi_{k}^{( \pm)}$можно выразить через многочлены Якоби $P_{n}^{(\alpha, \beta)}(z)$, см. $[1,10.8]$, где $z=(y+x) /(y-x)$ :

$$
\begin{aligned}
& \Psi_{k}^{(-)}(x, y)=(-1)^{k-r} d_{k} \cdot(y-x)^{2 l} P_{k-r}^{(2 r, 0)}(z), \\
& \Psi_{k}^{(+)}(x, y)=(-1)^{2 r} d_{k} \cdot(y-x)^{2 m} P_{k+r}^{(0,-2 r)}(z) .
\end{aligned}
$$

В частности, при $r=0$, т. е. при $l=m$, обе эти функции совпадают с точностью до знака. Тогда их можно выразить через многочлены Лежандра $P_{k}(z)$, см. $[1,10.10]$ :

$$
\Psi_{k}^{(+)}(x, y)=d_{k} \cdot(y-x)^{2 m} P_{k}(z) .
$$

Сферическую функцию $\Psi_{k}^{( \pm)}$можно рассматривать как обобщенную функцию на $V_{l} \otimes V_{m}$ с помощью билинейной формы $\langle\cdot, \cdot\rangle_{l m}$. Ее значение на функции $f \in V_{l} \otimes V_{m}$ равно значению вектора $\theta_{k}$ на компоненте Фурье этой функции:

$$
\left\langle\Psi_{k}^{( \pm)}, f\right\rangle_{l m}=\left\langle\theta_{k}^{( \pm)}, F_{k} f\right\rangle_{k}
$$

Ядро $\Pi_{k}(u, v ; x, y)$ получается из сферических функций $\Psi_{k}^{(-)}$и $\Psi_{k}^{(+)}$сдвигом с помощью матриц $g_{x y}$ и $g_{y x}$ соответственно:

$$
\begin{aligned}
& \Pi_{k}(u, v ; x, y)=(y-x)^{2 m}\left(T_{l m}\left(g_{x y}\right) \Psi_{k}^{(-)}\right)(u, v), \\
& \Pi_{k}(u, v ; x, y)=(y-x)^{2 l}\left(T_{l m}\left(g_{y x}\right) \Psi_{k}^{(+)}\right)(u, v) .
\end{aligned}
$$

Специализация $x=0, y \rightarrow \infty$ превращает матрицу $g_{x y}$ в единичную матрицу $E$; поэтому в силу (3.15) и (3.14) сферические функции получаются из ядра $\Pi_{k}$ предельным переходом:

$$
\begin{array}{r}
\lim _{y \rightarrow \infty} y^{-2 m} \cdot \Pi_{k}(u, v ; 0, y)=\Psi_{k}^{(-)}(u, v), \\
\lim _{x \rightarrow \infty}(-x)^{-2 l} \cdot \Pi_{k}(u, v ; x, 0)=\Psi_{k}^{(+)}(u, v) .
\end{array}
$$

Таким образом, формула обращения (3.12), или (3.14), равносильна следующим разложениям по сферическим функциям:

$$
\sum_{k} \frac{1}{\omega_{k}} \Psi_{k}^{(-)}(x, y)=(-x)^{2 l}, \quad \sum_{k} \frac{1}{\omega_{k}} \Psi_{k}^{(+)}(x, y)=(-1)^{2 r} y^{2 m} .
$$




\section{§4. Дифференциально-разностное уравнение для ядра Пуассона}

Алгебра Ли надгруппы $\widetilde{G}$ (надалгебра) есть прямая сумма $\widetilde{\mathfrak{g}}=\mathfrak{g}+\mathfrak{g}$, а алгебры Ли $\mathfrak{g}^{d}, \mathfrak{g}_{1}, \mathfrak{g}_{2}$ подгрупп $G^{d}, G_{1}, G_{2}$ состоят соответственно из пар $(X, X)$, $(X, 0),(0, X)$, где $X \in \mathfrak{g}$.

В этом параграфе мы находим действие операторов Ли $T_{l m}(X, Y), X, Y \in \mathfrak{g}$, на ядро Пуассона $P_{k}(x, y ; t)$, см. $(3.2)$, как на функцию от $x, y$ (так что тогда $t$ - параметр). Для пар $(X, X)$ ответ прост:

$$
T_{l m}(X, X) P_{k}=-T_{k}(X) P_{k},
$$

в правой части $T_{k}(X)$ действует на $P_{k}$ как на функцию от $t$. Эта формула есть по-другому записанная формула (3.3). Поэтому достаточно брать пары $(X, Y)$ из какого-нибудь подпространства в $\widetilde{\mathfrak{g}}$, дополнительного к $\mathfrak{g}^{d}$. В качестве такового мы возьмем подалгебру $\mathfrak{g}_{2}$.

Введем следующие дифференциальные операторы $S_{k}(X), k \in \mathbb{N}$, и $E(X)$ по переменной $t$, линейно зависящие от $X \in \mathfrak{g}$, которые на базисных элементах (1.1) задаются формулами

$$
\begin{aligned}
& E\left(L_{-}\right)=1, \quad S_{k}\left(L_{-}\right)=\frac{d^{2}}{d t^{2}}, \\
& E\left(L_{1}\right)=t, \quad S_{k}\left(L_{1}\right)=t \frac{d^{2}}{d t^{2}}-(2 k+1) \frac{d}{d t}, \\
& E\left(L_{+}\right)=t^{2}, \quad S_{k}\left(L_{+}\right)=t^{2} \frac{d^{2}}{d t^{2}}-2(2 k+1) t \frac{d}{d t}+(2 k+1)(2 k+2) .
\end{aligned}
$$

Лемма 4.1. Справедливы следующие соотношения коммутации:

$$
\begin{gathered}
S_{k}([X, Y])=T_{k}(X) S_{k}(Y)-S_{k}(Y) T_{k+1}(X), \\
E([X, Y])=T_{k}(X) E(Y)-E(Y) T_{k-1}(X) .
\end{gathered}
$$

Лемма доказывается непосредственной проверкой.

Лемма 4.2. Операторы $S_{k}(X)$ отображают $V_{k+1}$ в $V_{k}$.

Лемма следует из действия операторов $S_{k}(X)$ на одночлены:

$$
\begin{aligned}
S_{k}\left(L_{-}\right) t^{s} & =s(s-1) \cdot t^{s-2}, \\
S_{k}\left(L_{1}\right) t^{s} & =s[s-(2 k+2)] \cdot t^{s-1}, \\
S_{k}\left(L_{+}\right) t^{s} & =[s-(2 k+1)][s-(2 k+2)] \cdot t^{s} .
\end{aligned}
$$

Лемма 4.3. Операторы $S_{k}(X)$ и $E(X)$ сопряжсены в следующем смысле:

$$
\left\langle S_{k}(X) \psi, \varphi\right\rangle_{k}=(2 k+2)^{(2)} \cdot\langle\psi, E(X) \varphi\rangle_{k+1},
$$

где $\varphi \in V_{k} \subset V_{k+1} u \psi \in V_{k+1}$.

Доказательство. Для $X=L_{-}$равенство (4.3) есть

$$
\left\langle\frac{d^{2}}{d t^{2}} \psi, \varphi\right\rangle_{k}=(2 k+2)^{(2)} \cdot\langle\psi, \varphi\rangle_{k+1} .
$$

Оно сразу вытекает из определения (1.3), см. также (1.6). Для оператора $T_{k}(X)$ сопряженным относительно формы $\langle\cdot, \cdot\rangle_{k}$ является оператор $\left(-T_{k}(X)\right)$, 
см. (1.5). Операторы $S_{k}(X)$ можно выразить через $T_{k}(X)$ :

$$
\begin{aligned}
S_{k}\left(L_{-}\right) & =T_{k}\left(L_{-}\right)^{2}, \\
S_{k}\left(L_{1}\right) & =T_{k}\left(L_{1}\right) T_{k}\left(L_{-}\right)-(k+1) T_{k}\left(L_{-}\right), \\
S_{k}\left(L_{+}\right) & =T_{k}\left(L_{+}\right) T_{k}\left(L_{1}\right)-2(k+1) T_{k}\left(L_{1}\right)+2(k+1)^{2} .
\end{aligned}
$$

Поэтому для оператора $S_{k}(X)$ сопряженным является оператор $\left(d^{2} / d t^{2}\right) \circ E(X)$. Комбинируя это с (4.4), получаем (4.3).

Приведем еще (хотя нам дальше это не понадобится) сопряженный оператор (один из вариантов) для умножения на произвольную степень переменного $t$ :

$$
\left\langle\psi, t^{p} \varphi\right\rangle_{k+p}=\frac{1}{(2 k+2 p)^{(2 p)}}\left\langle\left(\frac{d}{d t}\right)^{p}\left(t \frac{d}{d t}-(2 k+p+1)\right)^{(p)} \psi, \varphi\right\rangle_{k} .
$$

Далее, введем следующие коэффициенты $\alpha_{k}, \beta_{k}, \gamma_{k}$ (для облегчения записи мы не будем явно указывать зависимость от $l, m)$ :

$$
\begin{aligned}
\alpha_{k} & =\frac{l+m-k}{(2 k+2)(2 k+1)}, \\
\beta_{k} & =-\frac{k(k+1)+(m-l)(l+m+1)}{2 k(k+1)}, \\
\gamma_{k} & =-\frac{(l+m+k+1)(k+m-l)(k-m+l)}{2 k(2 k+1)} .
\end{aligned}
$$

Теорема 4.4. Пусть $X \in \mathfrak{g}$. Onератор $T_{l m}(0, X)$ действует на ядро Пуассона $P_{k}(x, y ; t)$ следующим образом:

$$
T_{l m}(0, X) P_{k}=\alpha_{k} \cdot S_{k}(X) P_{k+1}+\beta_{k} \cdot T_{k}(X) P_{k}+\gamma_{k} \cdot E(X) P_{k-1}
$$

в левой части оператор действует на функцию от $x, y$, а в правой части операторь действуют на функиии от $t$.

Доказательство. Сначала возьмем $X=L_{-}$. Тогда равенство (4.8) имеет вид

$$
\frac{\partial}{\partial y} P_{k}=\alpha_{k} \cdot \frac{\partial^{2}}{\partial t^{2}} P_{k+1}+\beta_{k} \cdot \frac{\partial}{\partial t} P_{k}+\gamma_{k} \cdot P_{k-1}
$$

Докажем, что это равенство справедливо с коэффициентами (4.5)-(4.7). Напомним, что $P_{k}$ дается формулой (3.2). Положим для краткости

$$
a=2 l-j+1, \quad b=2 m-j+1, \quad \xi=x-t, \quad \eta=y-t .
$$

Вычисляя в (4.9) частные производные и производя сокращения, получаем равенство двух однородных многочленов от $\xi, \eta$ степени 2 :

$$
\begin{aligned}
& \xi\{(b-1)(\eta-\xi)+j \eta\} \\
= & \alpha_{k} \cdot\left\{b^{(2)} \xi^{2}+2 a b \xi \eta+a^{(2)}\right\}-\beta_{k} \cdot(\eta-\xi)\{(a-1) \eta+(b-1) \xi\}+\gamma_{k} \cdot(\eta-\xi)^{2} .
\end{aligned}
$$


Из него последовательно находим $\alpha_{k}, \beta_{k}, \gamma_{k}$ :

$$
\begin{gathered}
\alpha_{k}=\frac{j}{(a+b)(a+b-1)}, \quad \beta_{k}=-\frac{(a+b) b-2 a}{(a+b)(a+b-2)}, \\
\gamma_{k}=-\frac{(a-1)(b-1)(a+b-1+j)}{(a+b-1)(a+b-2)} .
\end{gathered}
$$

Подставляя сюда (4.10), получаем (4.5)-(4.7).

Для $X=L_{1}$ и $X=L_{+}$используем доказанное равенство (4.8) с $X=L_{-}$и соотношения коммутации - последовательно первое и второе из (1.2) и соответствующие соотношения для операторов $S_{k}(X)$ и $E(X)$ из (4.1) и $(4.2)$.

\section{§5. Взаимодействие преобразований Пуассона и Фурье с надалгеброй Ли}

В этом параграфе мы находим явные выражения для композиций операторов $T_{l m}(X, Y)$ и преобразований Пуассона и Фурье. Как и в $\S 4$, мы ограничимся подалгеброй $\mathfrak{g}_{2}$.

Теорема 5.1. Пусть $X \in \mathfrak{g}$. Onератор $T_{l m}(0, X)$ взаимодействует с преобразованием Пуассона $M_{k}$ следующим образом:

$$
T_{l m}(0, X) M_{k}=\alpha_{k}^{\prime} \cdot M_{k+1} E(X)+\beta_{k}^{\prime} \cdot M_{k} T_{k}(X)+\gamma_{k}^{\prime} \cdot M_{k-1} S_{k-1}(X),
$$

əəe

$$
\alpha_{k}^{\prime}=(2 k+2)^{(2)} \cdot \alpha_{k}, \quad \beta_{k}^{\prime}=-\beta_{k}, \quad \gamma_{k}^{\prime}=\frac{1}{(2 k)^{(2)}} \cdot \gamma_{k} .
$$

Доказательство. Возьмем $\varphi \in V_{k}$ и вычислим $T_{l m}(0, X) M_{k} \varphi$. Используем определение преобразования $M_{k}$, см. (3.4), и теорему 4.3:

$$
\begin{aligned}
T_{l m}(0, X) M_{k} \varphi & =T_{l m}(0, X)\left\langle P_{k}, \varphi\right\rangle_{k} \\
& =\alpha_{k} \cdot\left\langle S_{k}(X) P_{k+1}, \varphi\right\rangle_{k}+\beta_{k} \cdot\left\langle T_{k}(X) P_{k}, \varphi\right\rangle_{k}+\gamma_{k} \cdot\left\langle E(X) P_{k-1}, \varphi\right\rangle_{k} .
\end{aligned}
$$

K первому слагаемому применяем формулу (4.3), к третьему - ее же с заменой $k$ на $k-1$, ко второму - формулу (1.5). В результате получаем

$$
\begin{gathered}
T_{l m}(0, X) M_{k} \varphi=\alpha_{k} \cdot(2 k+2)^{(2)}\left\langle P_{k+1}, E(X) \varphi\right\rangle_{k}-\beta_{k} \cdot\left\langle T_{k}(X) P_{k}, \varphi\right\rangle_{k} \\
+\gamma_{k} \cdot \frac{1}{(2 k)^{(2)}}\left\langle P_{k-1}, S_{k-1}(X) \varphi\right\rangle_{k-1},
\end{gathered}
$$

а это есть (5.1). Заметим, что последнее слагаемое здесь корректно, поскольку $S_{k-1}(X) \varphi$ принадлежит $V_{k-1}$, см. лемму 4.2 .

Применим формулу (5.1) с $X=L_{1}$ к функции $\theta_{k}^{( \pm)}$. В левой части мы получим многочлен $T_{l m}\left(0, L_{1}\right) \Psi_{k}^{( \pm)}$из $V_{l} \otimes V_{m}$, собственный для операторов $T_{k}(h)$, $h \in H$, с собственным значением $\lambda^{ \pm 2 r}$, а сама формула (5.1) превращается в разложение этого многочлена по сферическим функциям $\Psi_{s}^{( \pm)}$:

$$
T_{l m}\left(0, L_{1}\right) \Psi_{k}^{( \pm)}= \pm\left\{\alpha_{k}^{\prime} \cdot \Psi_{k+1}^{( \pm)}+r \beta_{k}^{\prime} \cdot \Psi_{k}^{( \pm)}+\left(r^{2}-k^{2}\right) \gamma_{k}^{\prime} \cdot \Psi_{k-1}^{( \pm)}\right\}
$$

Коэффициенты разложения можно получить, умножая скалярно с помощью $\langle\cdot, \cdot\rangle_{l m}$ эти разложения на функции $\Psi_{s}^{(\mp)}$. 
Теорема 5.2. Пусть $X \in \mathfrak{g}$. Оператор $T_{l m}(0, X)$ взаимодействует с преобразованием Фурье $F_{k}$ следующим образом:

$$
F_{k} T_{l m}(0, X)=-\alpha_{k} \cdot S_{k}(X) F_{k+1}-\beta_{k} \cdot T_{k}(X) F_{k}-\gamma_{k} \cdot E(X) F_{k-1} .
$$

Доказательство. Пусть $f \in V_{l} \otimes V_{m}$. По формулам (3.5) и (1.5)

$$
F_{k} T_{l m}(0, X) f=\left\langle T_{l m}(0, X) f, P_{k}\right\rangle_{l m}=-\left\langle f, T_{l m}(0, X) P_{k}\right\rangle_{l m} .
$$

Подставим сюда (4.8). Операторы $S_{k}(X), T_{k}(X)$ и $E(X)$ можно вынести за знак формы $\langle\cdot, \cdot\rangle_{l m}$, поскольку они действуют на переменную $t$. В результате мы получаем (5.2).

\section{ЛитературА}

[1] Г. Бейтмен, А. Эрдейи, Высшие трансцендентные функиии. Функиии Бесселя, функции параболического иилиндра, ортогональные многочлены, Наука, М., 1966.

[2] В. Ф. Молчанов, Канонические представления и надгруппы для гиперболоидов, Функц. анализ и его прил., 39:4 (2005), 48-61.

[3] Ю. А. Неретин, Действие надалгебры в планшерелевском разложении и операторы сдвига в мнимом направлении, Изв. РАН, сер. матем., 66:5 (2002), 171-182.

[4] А. М. Переломов, Обобщенные когерентные состояния и их применения, Наука, M., 1987.

[5] H. Cohen, Sums involving the values at negative integers of L-functions of quadratic characters, Math. Ann., 217:3 (1975), 271-285.

[6] V. F. Molchanov, Canonical representations and overgroups, in: Lie Groups and Symmetric Spaces, Amer. Math. Soc. Transl., Ser. 2, vol. 210, Amer. Math. Soc., Providence, RI, 2003, 213-224.

[7] V. F. Molchanov, Canonical representations and overgroups for hyperboloids of one sheet and Lobachevsky spaces, Acta Appl. Math., 86:1-2 (2005), 115-129.

[8] V. F. Molchanov, Canonical representations on Lobachevsky spaces: an interaction with an overalgebra, Acta Math. Appl., 99:3 (2007), 321-337.

[9] N. Mukunda, Unitary representations of the homogeneous Lorentz group in an $O(2,1)$ basis, J. Math Phys., 9:1 (1968), 50-61.

[10] R. A. Rankin, The construction of automorphic forms from the derivatives of a given form, J. Indian Math. Soc., 20 (1956), 103-116.

Тамбовский государственный университет им. Г. Р. Державина e-mail: v.molchanov@bk.ru
Поступила в редакцию 31 октября 2014 г. 